\title{
Gestion optométrique d'une paralysie du troisième nerf crânien dans un milieu carcéral
}

Benoît Tousignant,

OD, M. Sc., MPH, FAAO

Professeur adjoint

École d'optométrie, Université de Montréal

\section{Résumé}

La paralysie du troisième nerf crânien (PTN) est un trouble neuro-ophtalmique que les optométristes peuvent rencontrer dans divers contextes cliniques. Elle affecte les muscles extraoculaires et peut nuire à divers degrés aux fonctions de la paupière et de la pupille. Chez les adultes, son étiologie est souvent associée à des lésions par compression du système nerveux central ou à des causes d'ischémie vasculaire découlant de maladies systémiques comme le diabète et l'hypertension. Le présent rapport de cas s'intéresse à la gestion d'une PTN dans un milieu carcéral canadien multidisciplinaire, milieu dans lequel la population de patients est souvent mal desservie. Il présente les pratiques actuelles en matière de neuro-imagerie pour les cas de PTN dans un contexte clinique conventionnel. Il aborde également les enjeux et avantages de la gestion de la PTN dans un milieu carcéral, en soulignant les particularités des soins aux patients vulnérables en milieu carcéral.

\section{MOTS CLÉS}

Paralysie du troisième nerf crânien, pénitencier, soins interdisciplinaires, neuro-imagerie, population mal desservie 


\section{INTRODUCTION}

La paralysie du troisième nerf crânien affecte les muscles extraoculaires, la paupière et la pupille et est un trouble rencontré par beaucoup d'optométristes. Le présent rapport de cas souligne les particularités de la gestion de ce trouble dans un milieu carcéral multidisciplinaire.

\section{RAPPORT DE CAS}

Un homme caucasien de 60 ans s'est présenté au service d'optométrie d'un pénitencier canadien de sécurité moyenne après avoir été aiguillé par le médecin de l'unité de santé mentale du pénitencier en raison d'une « vision réduite ou double ».

En réponse aux questions, le patient s'est surtout plaint d'avoir une vision floue depuis qu'il a perdu s'est lunettes. Il a nié la présence de diplopie, de douleur, de maux de tête et de traumatisme oculaire. Les réponses du patient étaient quelque peu limitées en raison de ses problèmes de santé mentale et d'un défaut de prononciation. Il a subi une ablation des cataractes en 2009 et porte habituellement des lunettes pour corriger un astigmatisme hypermétropique composé depuis 2011. La vision binoculaire et la santé oculaire ne présentaient par ailleurs aucune particularité.

Son historique médical actuel incluait le diabète de type 2 (1999, récente HbAlc 10,1 \%), l'obésité, l'hypertension, l'hypercholestérolémie, l'hyperplasie prostatique bénigne, la neuropathie diabétique, l'apnée du sommeil, le bégaiement, l'anémie, une légère déficience intellectuelle et la schizophrénie paranoïde. Il était traité avec de la gabapentine, de l'olanzapine, du valproate, de l'atorvastatine, de l'ézétimibe, du furosémide, de l'irbésartan, de la nifédipine, de l'insuline, du sulfate ferreux et de la térazosine. Ni l'historique familial médical ni l'historique familial oculaire n'étaient disponibles.

L’examen externe a révélé une ptose complète à l'œil droit, qui n’avait pas été mentionnée par le patient ni le médecin. Les acuités visuelles de loin non corrigées étaient de 6/21 OD (en tenant la paupière ouverte) et de 6/12 OS, avec un test de sténopé peu concluant. La distance manifeste de réfraction a démontré une ordonnance hypermétropique composée stable, avec des acuités visuelles corrigées de 6/6 OD (en tenant la paupière ouverte) et de 6/7,5 OS. Le test sous écran était peu concluant (compréhension et fixation difficiles), mais l'alignement oculaire par les réflexes de Krimsky a démontré un dioptre d'exotropie de 30 prismes et un dioptre d'hypotrophie de 10 prismes à l'œil droit. L'œil droit montrait des limitations de grade -4 pour l'adduction, l'abaissement et l'élévation, tandis l'œil gauche montrait une amplitude complète de la mobilité dans toutes les directions. Les pupilles étaient égales, réagissaient à la lumière et avaient une accommodation, sans déficit pupillaire afférent. L'examen du segment antérieur a révélé une ptose complète à l'œil droit, mais aucune autre particularité. La pression intraoculaire était de $14 \mathrm{mmHg}$ dans les deux yeux. La lentille intraoculaire gauche avait une légère opacification de la capsule postérieure. L'examen du fond de l'œil sous mydriatique n'a révélé aucune preuve de rétinopathie diabétique. Le champ visuel automatisé n’était pas disponible, et les champs visuels par confrontation étaient peu fiables.

Ce portrait clinique suggère une PTN complète, isolée avec une pupille fixe, à la lumière des éléments suivants : unilatérale, indolore, atraumatique, apparition récente d'une exophorie et hypotrophie, ptose complète, patient âgé de plus de 50 ans, fonction pupillaire normale et fonction normale des nerfs crâniens quatre et six. Le diabète est considéré être la cause la plus probable, compte tenu du profile glycémique du patient et de son hypertension systémique qui est sous contrôle. Le médecin du pénitencier a été consulté, et il a demandé une consultation en neuroimagerie afin d'exclure la possibilité d'étiologies par compression. Le personnel infirmier a été avisé de surveiller le patient quoditiennement pendant la semaine suivante pour détecter tout développement tardif d'une dilatation de la pupille droite. Un rendez-vous de suivi en optométrie a été fixé trois semaines plus tard. De nouvelles lunettes ont été commandées, mais aucune gestion de diplopie n'a été jugée nécessaire en raison de la ptose complète. Le patient a reçu des conseils quant à l'importance du contrôl de la glycémie et aux effets des maladies oculaires imputables au diabète. Il a été demandé à la nutritionniste du pénitencier d'intervenir afin d'insister sur les recommandations alimentaires. À l'examen de suivi, le patient était plus alerte, ce qui a facilité l'anamnèse. En réponse aux questions, il a nié avoir de la diplopie et de la douleur et semblait heureux de la nouvelle correction de la vue. Les résultats de neuro-imagerie n'étaient pas encore disponibles. Le dossier du patient indiquait qu'il n'y avait pas de changements de fonction de pupille. Les acuités visuelles corrigées étaient 6/6 OD et de 6/7,5 OD. L'examen externe a démontré qu'il n'y avait pas de ptose. Les yeux du patient étaient maintenant alignés lors de la fixation primaire du regard, avec un dioptre d'exotropie de 30 prismes de loin et de près, une vision binoculaire normale (filtre rouge) et une stéréopsie. Les mobilités extraoculaires avaient une amplitude complète dans toutes les directions. Les réflexes pupillaires étaient normaux, sans anisocorie. Un examen de suivi a été planifié deux mois plus tard. Le patient a été 
avisé d'alerter le personnel infirmier de toute diplopie ou tout changement dans sa vision ou apparence de sa pupille ou de sa paupière.

À l'examen de suivi deux mois plus tard, le patient a déclaré n'avoir observé aucun changement de son état. Les acuités visuelles corrigées étaient stables, et l'examen externe était normal. Le patient présentait maintenant une orthophorie de loin et de près. Les mobilités extraoculaires avaient une amplitude complète dans toutes les directions, et les réflexes pupillaires étaient normaux. Le rapport de radiologie incluait une tomodensitométrie de la tête et des orbites, avec contraste, qui montrait qu'il n'y avait pas de lésions intracrâniennes ou d'anomalies significatives. Le patient a été informé de son rétablissement complet en apparence et a été avisé de revenir dans deux ans, conformément à la politique du Service correctionnel du Canada à propos de la fréquence des examens ophtalmologiques.

\section{DISCUSSION}

\section{Présentation clinique et pathophysiologie}

Le troisième nerf crânien innerve les muscles supérieur, inférieur et droits internes, de même que l'oblique inférieur et releveur de la paupière. Ses fibres externes et dorsolatérales contrôlent l'accommodation et la myosis. Ainsi, la PTN se présente habituellement avec une ptose ipsilatérale, une hypotrophie avec exotropie concomitante, diplopie, et une possible pupille fixe et mydriatique, et une vision floue ${ }^{1,2}$. Une PTN complète inclut l'incapacité à à élever, baisser ou d'exercer une adduction de l'œil et est accompagnée d'une ptose complète. L'implication de la pupille dépend de l'étiologie. Une PTN complète ou partielle restreindra à divers degrés les mobilités extraoculaires en compromettant un ou plusieurs muscles extraoculaires et cause des implications pupillaires variables. Les paralysies partielles affectent le plus souvent soit la branche inférieure du nerf (muscle droit médial de l'œil, muscle droit inférieur de l'œil et le muscule oblique inférieur) ou sa branche supérieure (muscle droit supérieur de l'œil et le releveur). ${ }^{1,3}$ La plupart des

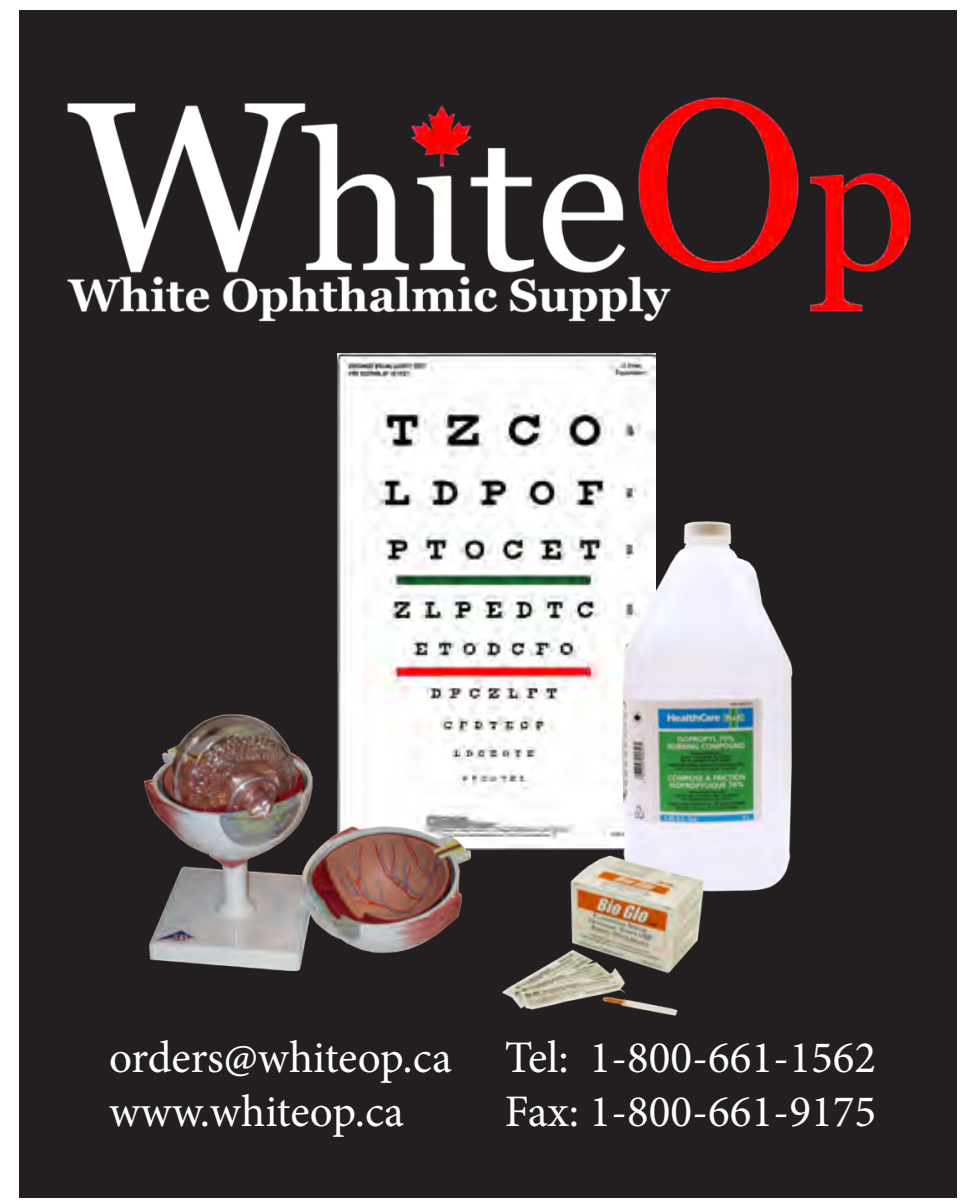


cas de PTN sont unilatéraux - les cas bilatéraux ont lieu seulement lorsque le noyau oculomoteur est impliqué.

Les étiologies principales des PTN incluent les causes vasculaires ou ischémiques (diabète, hypertension, maladie athérosclérotique), la compression (anévrisme, tumeur), les traumatismes et les causes congénitales ${ }^{1,3,4}$. L'étiologie est déterminée par les facteurs qui incluent l’implication de la pupille, la douleur, les facteurs de risque vasculaires et l'âge. Habituellement, la mydriase indiquera une lésion par compression ${ }^{1,3}$. Le nerf chemine du mésencéphale à l'orbite par l'espace sous-arachnoïdien, il passe à côté du polygone de Willis, près de l'artère communicante postérieure, là où un anévrisme peut compresser les fibres externes pupillaires, entraînant une mydriase et une perte de la fonction pupillaire. Les paralysies par compression sont souvent accompagnées de douleur ou maux de tête ${ }^{3,4}$. Inversement, les causes ischémiques n'affecteront généralement que les fibres internes du troisième nerf crânien résultant en une pupille fixe indolore ${ }^{4,5}$. Chez les patients âgés de plus de 50 ans, il est plus probable que la PTN soit de nature ischémique, où le vasa nervorum peut souffrir d'ischémie, affectant ainsi les fibres internes qu'il alimente. Les patients de moins de 50 ans sont plus susceptibles de souffrir d'étiologies compressives ${ }^{1}$. La PTN peut être combinée à des paralysies du quatrième, cinquième ou sixième nerfs crâniens avec des lésions affectant le sinus caverneux ${ }^{6}$.

\section{Diagnostic différentiel}

Dans le présent cas, le diagnostic différentiel inclut la myasthénie grave, la PTN partielle et une déviation pré-existante ou décompensée avec une ptose concomitante. Ce dernier a été exclu sur la base des dossiers médicaux antérieurs qui montraient un alignement oculaire normal et une fonction normale de la paupière. Une paralysie partielle a été exclue compte tenu de l'handicap complet de la paupière et des muscles extraoculaires. Pour terminer, une myasthénie oculaire grave peut être présente avec de la diplopie et toucher les muscles extraoculaires ou les muscles releveurs. Cependant, les déséquilibres musculaires augmenteront généralement avec la fatigue musculaire. Cela a été exclu dans le cas présent puisque la ptose était complète et constante, l'adduction et l'élévation étant complètement altérées.

\section{Gestion du cas}

La période de rétablissement pour une PTN dont les causes suspectées sont de nature vasculaire est habituellement entre trois et 12 mois $^{1,3}$. Sa gestion implique de surveiller les symptômes de diplopie, de surveiller l'implication pupillaire, de réaliser une neuro-imagerie etde cibler la condition systémique sous-jacente ${ }^{3,4}$. La gestion de la dip-

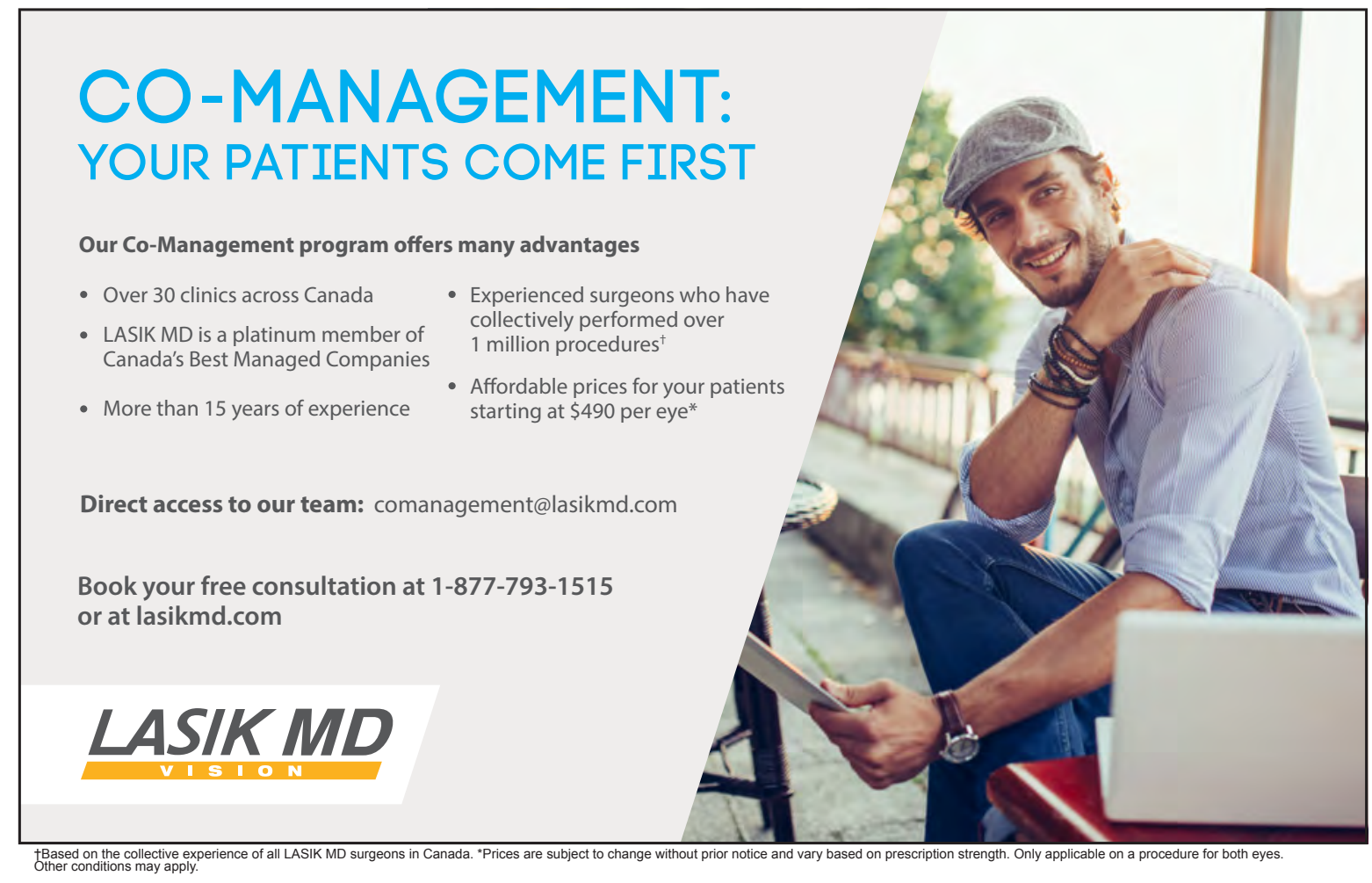


lopie exige l'application d'un timbre ou l'utilisation de lentilles givrées ou de prismes de Fresnel pour réduire les symptômes $^{3,4}$. Si les prismes de Fresnel sont simples et abordables, les lentilles givrées sont coûteuses et possèdent de longs délais de livraison, alors qu'il s'agit probablement d'une solution temporaire. Dans le présent cas, bien que l'anamnèse était peu fiable, la présence d'une ptose complète permettait d'éliminer des affections potentielles de diplopie. De plus, lorsque la ptose s'est atténuée, le patient a également retrouvé une vision binoculaire.

Comme certaines causes compressives se présentent initialement avec une fonction pupillaire normale, une pupille fixe d'une PTN ischémique présumée doit être suivie de près pendant la première semaine pour vérifier l'apparition d'implication pupillaire ${ }^{1,3,6}$. Dans le présent cas, la surveillance a été assurée par le personnel infirmier qui était présent tous les jours.

\section{Rôle de la neuro-imagerie}

Les données probantes fondées sur la neuro-imagerie quant à la PTN ischémique présumée chez les patients de plus de 50 ans a évolué au fil du temps et demeure controversée ${ }^{7,8}$. Le problème principal concerne la valeur diagnostique de la neuro-imagerie pour éliminer l'anévrisme ou d'autres lésions par compression comme causes de tels cas. Les pratiques actuelles en ophtalmologie ont évolué de l'observation minutieuse et attentive (de 3 à 6 mois) à la réalisation d'imagerie par résonnance magnétique (IRM) et d'angiographie par résonnance magnétique (ARM) pour la plupart des patients ayant une PTN, de manière à éliminer le plus efficacement la possibilité de lésions par compression ${ }^{6,9-11}$. Malgré un manque actuel de données probantes de haute qualité (revue systématique, méta-analyses ou directives cliniques fondées sur les données cliniques) à ce sujet, les études prospectives ${ }^{10,11}$ ont démontré que, dans $14 \%$ à 16,5\% des cas de patients de plus de 50 ans ayant une PTN qui était présumée avoir des étiologies vasculaires, les causes de la PTN étaient autres en réalité (lésions par compression, anévrismes, maladie démyélinisante, etc.), et que la neuro-imagerie (tomodensitométrie et plus récemment IRM et ARM) a mené à une gestion appropriée plus rapide de la cause sous-jacente. Cependant, le faible ratio coût-bénéfice de la neuro-imagerie par IRM pour tous les patients peut être un facteur limitant. Par conséquent, certains cliniciens peuvent utiliser la tomodensitométrie et l'angiographie par tomodensitométrie (AT) qui sont moins dispendieuses que l'IRM, mais qui peuvent tout de même détecter les lésions par compression et les lésions vasculaires ${ }^{12}$. Dans le cas présent, la conviction du médecin quant à la probabilité d'une cause ischémique a mené à une tomodensitométrie, vraisemblablement motivée par des considérations de coût dans un contexte de soins subventionnés par le public. Des praticiens attentionnés devraient donc demander des IRM (avec ARM) pour tous les patients ayant une PTN, même si l'étiologie ischémique est présumée, de même que pour les cas susceptibles d'être causés par compression ou par traumatisme (implication pupillaire [anisocorie supérieure à $2 \mathrm{~mm}$ ], déficit partiel de la mobilité ou mois de 50 ans) ${ }^{9-11}$.

\section{Implications de la pratique optométrique en milieu carcéral}

Beaucoup de praticiens connaissent mal la pratique optométrique en milieu carcéral. Au Canada, le SCC engage des optométristes dans le cadre de leurs obligations de soins aux contrevenants, en vue de préserver la santé oculaire, de faciliter les tâches quotidiennes, de favoriser les programmes d'éducation et de réhabilitation et ultimement la réinsertion sociale. Cette pratique a des enjeux et des avantages distincts.

La population en milieu carcéral est mal desservie, avec un fardeau significatif de maladies. Les niveaux élevés de maladie systémique et les facteurs de risque (p. ex. diabète, hypertension, hypercholestérolémie, utilisation de drogue et d'alcool, tuberculose, hépatite $\mathrm{C}$, etc.) mènent à un nombre significatif de complications du diabète, neuropathie optique, cataractes, rétinopathies, etc. Les aiguillages vers des spécialistes à l'externe (p. ex. ophtalmologie, neuro-imagerie) peuvent être soumis à des limitations (disponibilité du transport, escortes de sécurité, etc.). Beaucoup de contrevenants ne désirent pas faire des voyages à l'extérieur du milieu carcéral puisque les conditions de transport impliquent des contraintes et des véhicules qui ne sont pas confortables. Le refus par un contrevenant de recevoir des soins médicaux (sauf lors d'exceptions spécifiques) et d'être référé à l'externe peut se traduire par la progression d'une maladie ce qui potentiellement peut augmenter la morbidité. En raison de dotations budgétaires limitées, un établissement de 400 à 500 contrevenants peut avoir accès à une clinique d'optométrie une journée par mois. Le nombre de patients vus en une journée (approximativement 6 à 14) est inversement proportionnel au niveau de sécurité de l'établissement. Ces facteurs mènent à des temps d'attente de 3 à 12 mois pour avoir accès à des services optométriques.

Les politiques du SCC relativement à l'accès des contrevenants aux soins optométriques sont énoncés dans Cadre national des services de santé essentiels du SCC $\mathrm{SC}^{13}$. À l'heure actuelle, les contrevenants sont autorisés à avoir un examen de la vue tous les deux ans et à avoir un remplacement de leurs lunettes (verres simples ou lunettes bifocales) payé par le 
SCC tous les trois ans. L'examen de la vue et les visites de suivi peuvent être plus fréquentes sur demande du médecin ou de l'optométriste de l'établissement, comme dans le cas présent. En raison de la demande importante et l'offre ponctuelle de services optométriques, le suivi de certaines conditions est plus difficile et engendre des retards dans la surveillance de certaines troubles, comme l'opacification de la capsule postérieure gauche du patient dans le cas présenté

Le SCC fournit des salles d'examen et de l'équipement ophtalmique. Bien que les salles d'examen sont destinées à permettre à un optométriste à exécuter des examens complets des yeux, l'auteur du présent texte a constaté par son expérience que l'équipement n'est pas distribué de manière égale entre les établissements. Bien que les exigences de base soient habituellement présentes (chaise ophtalmique, réfracteur, autoréfractomètre, lampe à fente [avec lentille de fond et le goniomètre à prisme], tonomètre, trousse de diagnostic de poche, trousse de lentilles d'essais, tests de vision binoculaire, ophtalmoscope indirect, etc.), l'équipement d'imagerie (photographie de fond et tomographie par cohérence optique [TCO]) manque cruellement dans les établissements. Quelques établissements ont des champs visuels automatisés. Bien que ce n'était pas possible dans le présent cas, une évaluation du champ visuel aurait été bénéfique, car une lésion par compression située le long de la voie optique peut être soulignée par divers types de scotomes. Des requêtes pour de l'équipement additionnel sont possibles et peuvent être acceptées, mais la décision repose entre les mains des autorités du SCC et est prise selon les dotations budgétaires et autres spécialités médicales. Au besoin, les consultations à l'externe peuvent être réalisées auprès d'un optométriste ou ophtalmologiste de la région, bien que ces requêtes soient assujetties aux mêmes contraintes concernant la disponibilité du transport et les escortes de sécurité.

L’environnement clinique dans le milieu carcéral apporte également des avantages distincts au clinicien en optométrie. Le dossier optométrique du patient est une partie du dossier médical complet du contrevenant, lequel inclut des fiches médicales, pharmaceutiques, dentaires, psychosociales, psychiatriques et nutritionnelles. Cela fournit un accès privilégié à de l'information complémentaire et à des résultats de test qui contribuent grandement à la qualité des soins optométriques. De plus, le personnel des services de santé des établissements est composé d'infirmières, qui ont des rôles élargis similaires à ceux des infirmières praticiennes, et est présent chaque jour et est souvent présent pendant la nuit. Ces infirmières sont des collaboratrices clés qui fournissent des soins complets aux patients. Les médecins de chaque établissement, de même que d'autres professionnels (pharmaciens, nutritionnistes) sont également disponibles pour la consultation. Cette accessibilité est bien illustrée dans le cas présent, car la co-gestion qui a été faite par l'optométriste et le médecin a mené à l'imagerie et la permanence du personnel infirmier a permis une surveillance quotidienne de la fonction pupillaire et plusieurs professionnels ont été impliqué à tenter de contrôler le diabète du patient.

\section{Remerciements}

L'auteur tient à remercier le Service correctionnel du Canada d'avoir autorisé l'accès au dossier du patient et aux équipes multidisciplinaires composées de professionnels dévoués qui aident à améliorer et préserver la santé des contrevenants.

\section{Références}

1. Akagi, T., K. Miyamoto, S. Kashii et N. Yoshimura. « Cause and prognosis of neurologically isolated third, fourth, or sixth cranial nerve dysfunction in cases of oculomotor palsy », Jpn J Ophthalmol, 2008; $\mathrm{n}^{\circ} 52$, p. 32-35.

2. Bruce, B., V. Biousse et N. Newman. « Third Nerve Palsies », Semin Neurol, 2007; $\mathrm{n}^{\circ}$ 27, p. 257-268.

3. Yanovitch, T., et E. Buckley. « Diagnosis and management of third nerve palsy », Curr Opin Ophthalmol, 2007; n 18, p. 373-378.

4. von Noorden, G.K., et E.C. Campos. Binocular Vision and Ocular Motility: Theory and Management of Strabismus, 6 édition. En ligne : http://www.cybersight.org/bins/content_page.asp? cid=1-2193 (consulté le 11 mars 2016).

5. Jung, J.S., et D.H. Kim. « Risk factors and prognosis of isolated ischemic third, fourth, or sixth cranial nerve palsies in the Korean population ", $J$ Neuroophthalmol, 2015; $\mathrm{n}^{\circ} 35$, p. 37-40.

6. Lo, C.-P., C.-F. Huang, C.-C. Hsu et coll. « Neuroimaging of isolated and non-isolated third nerve palsies », Br J Radiol, 2012; n 85, p. 460-467.

7. Volpe, N.J., et A.G. Lee. « Do patients with neurologically isolated ocular motor cranial nerve palsies require prompt neuroimaging? », J Neuroophthalmol, 2014; $\mathrm{n}^{\circ} 34$, p. 301-305.
8. Tamhankar, M.A., et N.J. Volpe. « Management of acute cranial nerve 3, 4 and 6 palsies: role of neuroimaging », Curr Opin Ophthalmol, 2015; n ${ }^{\circ} 26$, p. 464-468.

9. Murchison, A.P., M.E. Gilbert et P.J. Savino. « Neuroimaging and acute ocular motor mononeuropathies: A prospective study », Arch Ophthalmol, 2011; $\mathrm{n}^{\circ} 129$, p. 301-305.

10. Tamhankar, M.A., V. Biousse, G.S. Ying et coll. « Isolated third, fourth, and sixth cranial nerve palsies from presumed microvascular versus other causes: a prospective study », Ophthalmology, 2013; $\mathrm{n}^{\circ} 120$, p. 2264-2269.

11. Chou, K.L., S.L. Galetta, G.T Liu et coll. « Acute ocular motor mononeuropathies: prospective study of the roles of neuroimaging and clinical assessment », J Neurol Sci, 2004; n 219, p. 35-39.

12. Mehta, S., L.A. Loevner, I. Mikityansky et coll. « The diagnostic and economic yield of neuroimaging in neuro-ophthalmology », $J$ Neuroophthalmol, 2012; $\mathrm{n}^{\circ}$ 32, p. 139-144.

13. Service correctionnel du Canada. Cadre national relatif aux soins de santé essentiels, 2015. En ligne : https://buyandsell.gc.ca/cds/public /2014/02/06/98ad3da3ellddf8a9f779fe07662329e/natessential_hsframework_april20131.pdf (consulté le 18 mars 2016). 